\title{
Supervision registers and medium secure units
}

\author{
Jim Isherwood
}

The introduction of supervision registers for mentally ill people according to the NHS Execultive Guidellines in February 1994 aroused debate among poychiatritsts. This paper highilghts many of the lssues and describes the development of the supervision register at one medium secure unit.

In her letter to the Secretary of State for Health, the President of the Royal College of Psychiatrists addressed a number of objections on behalf of the College. These included likely increased demands on clinicians' time, disincentives for professionals to become involved with this vulnerable group of patients, fears of criticism, breached confidentiality and an adverse effect on therapeutic relationships (Caldicott, 1994).

Further concerns include the possibility of time consuming and expensive judicial scrutiny of decisions or litigation (Harrison \& Bartlett, 1994). It has also been suggested that the stigmatising effect of registration may make primary services more inaccessible for difficult to treat patients. Moreover, the inner urban area may struggle to provide more services where the Care Programme Approach (CPA) is already used to good effect (Holloway, 1994).

Support for the supervision register has come from the government and the medical profession. In her reply to Dr Caldicott, the former Secretary of State, Mrs Bottomley emphasised the government's commitment to giving the highest priority for care and treatment to those patients most at risk. She rejected the criticisms of the Royal College and denied that a considerable proportion of psychiatric patients would be involved. Mrs Bottomley conceded that clinicians may be called upon to justify their decisions but did not foresee an increase in their legal and ethical responsibilities (Secretary of State for Health, 1994).

Psychiatrists have been blamed for relying on the "mandarins and boffins of the Department of Health" to tell them how to identify the severely mentally ill and provide for their needs (Tyrer \& Kennedy, 1995). These authors propose the supervision register as a powerful tool in negotiation for a proper level of investment and argue that clinicians will not be scapegoated. Furthermore they claim that the register will provide the only way of tracking itinerants across district borders.

It has been recognised by the NHS Trusts and Health Commissions of south-east London that it would not be possible to fully implement the guidelines (McCarthy et al, 1995). The combination of an inner city population, inadequate resources and increasing numbers of seriously disturbed patients threatened to compromise patient care. The resulting agreement produced a register consisting only of patients with a diagnosis of major mental illness and a history of violence or serious dangerousness to others (although all conditionally discharged patients were included).

\section{Background}

Ravenswood House is the medium secure unit for the former Wessex reglon situated in the southeast corner of that region. The population is largely rural with centres in Southampton and Portsmouth. Most patients are discharged from Ravenswood House to their district of origin although some choose to settle nearby. Occasionally patients require long-term psychiatric followup from the forensic service and this is provided by the community forensic psychiatric team based at the unit. Such patients have all been discharged from the unit and reside in the Southampton/Portsmouth area. The team is led by a consultant forensic psychiatrist who may supervise a senior registrar. Other permanent members include a forensic community psychiatric nurse (CPN) and an approved social worker dedicated to the medium secure unit. Contributions from the psychology, behaviour therapy and occupational therapy departments are readily available. Patients are seen regularly by the CPN, and at intervals not exceeding two months by the whole team at the weekly ward round. All patients are subject to the CPA and included on the CPA register.

The essential requirements of the supervision register are set out in Health Service Guidelines (NHS Management Executtve, 1994) but its operation may be approached in different ways. Discussions at Ravenswood House produced 
some key questions that were considered vital in developing the register and so it was decided to consult other reglonal secure units to discover any common practices.

\section{The study}

In October 1994 anonymous questionnaires were sent to each regional secure unit (RSU) in England and Wales. There were three closed questions and one open question as follows:

(1) In view of the many objections to supervision registers, have you or do you intend to have a register?

(2) Do you intend to share a register with other provider units or keep one solely for the medium secure unit?

(3) Will you register only patients directly supervised by your own unit or include patients referred for opinions by others, e.g. catchment area psychiatrists?

(4) How do you intend to maintain confidentiality where this is appropriate, e.g. "evidence of dangerous behaviour or offending"?

\section{Findings}

Fifteen replies were received from 19 questionnaires.

\section{Registers}

Thirteen RSUs intended to have a register but one of these planned only to register those at significant risk of severe violence to others. Of the two without a register, one had decided that registration would remain the responsibility of the local service. The Welsh Office had no plan to introduce the register to Wales.

\section{RSU only or shared?}

Eight RSUs intended to share a register within their NHS Trust. Four chose to maintain their own and one had yet to decide.

\section{Direct supervision only?}

Where policy had been agreed, 10 units would only register patients that were directly supervised by them. Only one intended to include other patients.

\section{Confidentiality}

Understandably, this question produced the greatest range of answers. There was almost universal concern that confidentiality would be breached. Several replies described limited access to the register or confirmed that contents were subject to the same rules of confidentiality as medical records. Only one unit had devised a mechanism to protect patient confidentiality. In this case, only the patient's name would be divulged to enquirers. Other information was restricted to medical staff.

\section{The Wessex approach}

After considering the above replies, a policy was agreed at Ravenswood House. A register is kept on site, distinct from other registers within the Trust. Only out-patients managed by the community forensic psychiatry team are included. Where appropriate, catchment area psychiatrists may be advised to include patients on their own supervision registers.

Access to the register may only be achieved via the Responsible Medical Officer (RMO) or most senior doctor on call. In the event of an enquiry only relevant information will be disclosed. It is envisaged that the patient's name, address and date of birth will suffice in all circumstances. In any case, should the mental health of these patients deteriorate in the community it is usual for assessment by staff from the unit to occur on the same day.

At present there are 12 patients managed by the community team. Of these, eight are conditionally discharged under Section 41 of the Mental Health Act and four are informal. Most patients are included on the supervision register, generally, but not always, because of a risk of serious violence to others.

\section{Comment}

The questionnaire was distributed to regional secure units during the early stage of the development of supervision registers. The response rate was very high. At that time the majority of English RSUs had begun to consider how to implement the Health Service Guidelines. There were different approaches to the problem reflecting different circumstances. Forensic psychiatrists had generally decided to register only those patients directly under their care. There was, and still is, overwhelming concern about confidentiality but few suggestions as to how this may be achieved. A number of respondents expressed strong views that it could not be achieved.

The situation in Wessex is perhaps more promising than elsewhere. The patient group is small and restricted to a limited geographical area. Supervision of these patients can be achieved by a cohesive multidisciplinary team which meets weekly. There has been a significant but manageable increase in workload for the team since the introduction of the Care Programme Approach but this has been appreciated 
by carers and professionals from the community who attend the meetings. The arrival of the supervision register had minimal effect on workload although time has been spent counselling and reassuring those patients who have been registered. In particular, patients who have lived in the community for several years have reacted most negatively and therapeutic relationships with them may have been strained. This picture may not be repeated in inner city areas where there will inevitably be greater demand on clinical time from a more itinerant population.

Any measure to improve patient care and follow-up in this vulnerable group must be supported but it is not yet clear that supervision registers will benefit patients more than the Care Programme Approach. Similarly, there is uncertainty as to how registration will enable services to follow patients who drift across boundaries if the patients themselves do not report their movements.

There has already been a report of a patient's access to community facilities prejudiced by registration (Quinn, 1995). This antipathy toward patients has not been evident so far in the Ravenswood House Community Forensic Team. Time will tell if this proves an isolated occurrence.

\section{References}

Caldicot,, F. (1994) Letter to the Secretary of State for Health. Psychiatric Bulletin, 18, 385-386.

HARRISON, G. \& BARTLETT, P. (1994) Supervision registers for mentally ill people. British Medical Joumal, s09, 551552.

Howoway, F. (1994) Supervision registers. Recent government policy and legislation. Psychiatric Bulletin. 18. 593-596.

MCCARTHY, A. Roy, D., HoLloway, F., et al (1995) Supervision registers and the care programme approach: a practical solution. Psychiatric Bullettr. 19. 195-199.

NHS MANAGEMENT EXECUTIVE (1994) Introduction of Supervision Registers for Mentally III People from 1 Aprll 1994. Leeds: NHSME (HSG(94)5).

QUINN, J. D. (1995) Supervision registers (letter). Psychiatric Bulletin, 19, 513.

SECRETARY OF STATE FOR HEALTH (1994) Letter for the President of the Royal College of Psychiatrists. Psychiatric Bulletin, 18, 387-388.

TYRER, P. \& KENNEDY, P. (1995) Supervision registers: a necessary component of good psychiatric practice. Psychiatric Bulletin, 19, 193-194.

Jim Isherwood, Senior Registrar in Forensic Psychiatry, Ravenswood House, Knowle Hospital, Fareham, Hampshire PO17 5NA 